\title{
Transverse resonance island buckets for synchrotron-radiation based electron time- of-flight spectroscopy
}

T. Arion, W. Eberhardt, J. Feikes, A. Gottwald, P. Goslawski, A. Hoehl, H. Kaser, M. Kolbe, J. Li, C. Lupulescu, M. Richter, M. Ries, F. Roth, M. Ruprecht, T. Tydecks, and G. Wüstefeld

Citation: Review of Scientific Instruments 89, 103114 (2018); doi: 10.1063/1.5046923

View online: https://doi.org/10.1063/1.5046923

View Table of Contents: http://aip.scitation.org/toc/rsi/89/10

Published by the American Institute of Physics

\section{Articles you may be interested in}

Real-time adjustable, $11 \mu \mathrm{s}$ FHM, $>5 \mathrm{kHz}$, piezo electric pulsed atomic beam source

Review of Scientific Instruments 89, 103115 (2018); 10.1063/1.5038013

Development of high-speed vacuum ultraviolet spectroscopy using a modified Seya-Namioka monochromator and channel electron multiplier detector in the HL-2A tokamak

Review of Scientific Instruments 89, 10D134 (2018); 10.1063/1.5037504

Plasma rotation measurement using UV and visible spectroscopy on Aditya-U tokamak Review of Scientific Instruments 89, 10D132 (2018); 10.1063/1.5039333

Methods of improving spatial resolution for IR spectroscopy in atmospheric-pressure plasma systems Review of Scientific Instruments 89, 10B114 (2018); 10.1063/1.5039389

X-ray backlighter requirements for refraction-based electron density diagnostics through Talbot-Lau deflectometry

Review of Scientific Instruments 89, 10G127 (2018); 10.1063/1.5039342

Time-tagged coincidence counting unit for large-scale photonic quantum computing

Review of Scientific Instruments 89, 103113 (2018); 10.1063/1.5044250

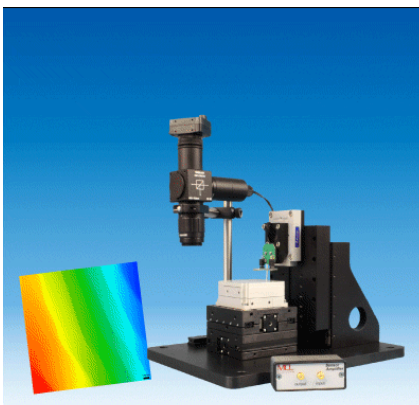

AFM \& NSOM
Nanopositioning Systems

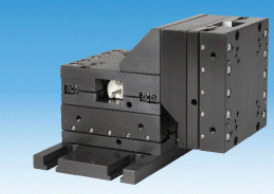

Micropositioning

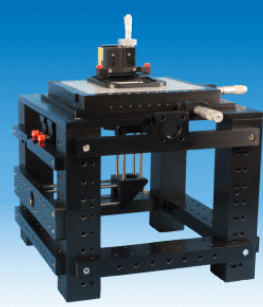

Single Molecule Microscopes 


\title{
Transverse resonance island buckets for synchrotron-radiation based electron time-of-flight spectroscopy
}

\author{
T. Arion, ${ }^{1}$ W. Eberhardt, ${ }^{1,2}$ J. Feikes,${ }^{3}$ A. Gottwald, ${ }^{4}$ P. Goslawski, ${ }^{3}$ A. Hoehl, ${ }^{4}$ H. Kaser, ${ }^{4}$ \\ M. Kolbe, ${ }^{4}$ J. Li, ${ }^{3}$ C. Lupulescu, ${ }^{2}$ M. Richter, ${ }^{4, a)}$ M. Ries, ${ }^{3, b)}$ F. Roth, ${ }^{5, c)}$ M. Ruprecht, ${ }^{3}$ \\ T. Tydecks, ${ }^{3}$ and G. Wüstefeld ${ }^{3}$ \\ ${ }^{1}$ Center for Free-Electron Laser Science/DESY, Notkestraße 85, D-22607 Hamburg, Germany \\ ${ }^{2}$ Institute of Optics and Atomic Physics, TU Berlin, Hardenbergstraße 36, 10623 Berlin, Germany \\ ${ }^{3}$ Helmholtz-Zentrum Berlin, Albert-Einstein-Straße 15, 12489 Berlin, Germany \\ ${ }^{4}$ Physikalisch-Technische Bundesanstalt, Abbestraße 2-12, 10587 Berlin, Germany \\ ${ }^{5}$ Institute for Experimental Physics, TU Bergakademie Freiberg, Leipziger Straße 23, 09599 Freiberg, Germany
}

(Received 3 July 2018; accepted 7 October 2018; published online 26 October 2018)

\begin{abstract}
At the Metrology Light Source (MLS), the compact electron storage ring of the PhysikalischTechnische Bundesanstalt (PTB) with a circumference of $48 \mathrm{~m}$, a specific operation mode with two stable closed orbits for stored electrons was realized by transverse resonance island buckets. One of these orbits is closing only after three turns. In combination with single-bunch operation, the new mode was applied for electron time-of-flight spectroscopy with an interval of the synchrotron radiation pulses which is three times the revolution period at the MLS of $160 \mathrm{~ns}$. The achievement is of significant importance for PTB's future programs of angular-resolved electron spectroscopy with synchrotron radiation and similar projects at other compact electron storage rings. The scheme applied here for selecting the photons originating from a particular orbit by optical imaging has been used before in fs slicing applications and may be relevant for the BESSY VSR project of the Helmholtz-Zentrum Berlin. Published by AIP Publishing. https://doi.org/10.1063/1.5046923
\end{abstract}

Current developments of accelerator-based (soft) X-ray sources for materials research refer to two main directions: (a) single-pass free-electron lasers (FELs) based on selfamplified spontaneous emission (SASE) ${ }^{1-5}$ or different seeding concepts ${ }^{6,7}$ and (b) diffraction-limited storage rings (DLSRs). ${ }^{8}$ FELs provide for single-user experiments' peak brightness femtosecond pulses with a repetition rate of $10^{2}$ up to a maximum of $10^{6} \mathrm{pulses} / \mathrm{s}$ in a special time-pattern to study ultra-fast dynamical and non-linear processes. DLSRs are optimized, on the other hand, for diffraction limited high average brightness synchrotron radiation (SR), i.e., for achieving the ultimate lateral spot size on a sample and, e.g., spectromicroscopy applications at pulse durations ranging from some 10 ps to more than 200 ps. The advantage of DLSRs and generally storage rings is the possibility of multi-user operation at repetition rates beyond $100 \mathrm{MHz}\left(10^{8} \mathrm{pulses} / \mathrm{s}\right.$ at regular spacing). Here, the samples can be interrogated multiple times in the linear response regime enabling to make "movies" of processes. While many storage ring facilities around the world are planning upgrades to achieve the diffraction limited photon beam, the BESSY VSR project at the Helmholtz-Zentrum Berlin $(\mathrm{HZB})^{9}$ follows an alternative concept. This upgrade of the electron storage ring BESSY II in Berlin-Adlershof is aimed at providing short photon pulses down to $2 \mathrm{ps}$ (rms) in parallel with multi-user standard (>15 ps) operation and even down to $400 \mathrm{fs}$ (rms) in a special operation mode.

\footnotetext{
a)Electronic mail: Mathias.Richter@ptb.de

b)Electronic mail: Markus.Ries@helmholtz-berlin.de

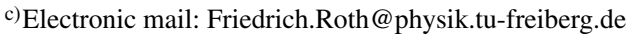

BESSY VSR is a novel approach to create photon pulses of different lengths and periods circulating simultaneously for all SR beamlines by using a pair of superconducting cavities shaping the longitudinal phase space. The optics at each beamline can be tuned to accept the light from the original orbit or from the deflected electron bunches enabling each individual user to freely switch, e.g., between high average photon flux for X-ray spectroscopy, microscopy, and scattering and picosecond pulses for dynamic studies. Preparatory work on the BESSY VSR concept has, however, been performed at the Metrology Light Source (MLS) of the PhysikalischTechnische Bundesanstalt (PTB), Germany's national metrology institute. ${ }^{10}$ The MLS represents a compact $630 \mathrm{MeV}$ electron storage ring with a circumference of $48 \mathrm{~m}$ in the immediate vicinity of BESSY II and is dedicated to SR based metrology. Also operated by HZB, the MLS is optimized regarding its regular performance (electron beam current and lifetime) as well as for special operations with variable electron energies and bunch lengths. By design, it is equipped with additional families of sextupole and octupole magnets and therefore is ideally suited to investigate nonlinear beam dynamics. ${ }^{10-13}$

In particular, the population of the so-called transverse resonance island buckets (TRIBs) has successfully been realized at the MLS, ${ }^{13}$ i.e., the simultaneous operation of two orbits. It offers the possibility to store two distinct transversely displaced electron beams, e.g., homogeneous fill and pseudo single bunch, separated in angle and space. Thus, apart from pulse-picking schemes, limited in some ways, the different bunch modes may be simply selected by means of optical imaging at individual SR beamlines, respectively, 
which may be relevant for BESSY VSR. Optical selection schemes have already been realized at fs-slicing sources at the Advanced Light Source (ALS) in Berkeley ${ }^{14}$ and at BESSY II. ${ }^{15,16}$ Pseudo-single-bunch as shown at the ALS presents another concept, whereas a single electron bunch is displaced transversely from the other electron bunches using a shortpulse, high-repetition-rate kicker magnet. ${ }^{17}$ For compact storage rings like the MLS, TRIB operation provides, however, a further promising aspect: a single bunch trapped in an island in the first turn will be observed in another island in the second turn and so forth. In the case of a 3rd order resonance, it will come back to the first island bucket again at the 4th turn. This property may be exploited to generate photon pulses with sub-revolution repetition rates. The latter allows, even at smaller rings with high revolution frequencies, electron time-of-flight (eTOF) spectroscopy to be performed. eTOF spectroscopy is usually applied in the singlebunch mode of a storage ring and based on the evaluation of electron kinetic energies from the interval between a SR pulse, triggered by the bunch-clock signal, and the electron detection. The corresponding start-stop measurements are, however, significantly disturbed if the electron times-of-flight of typically up to about $400 \mathrm{~ns}$ exceed the photon pulse interval.

In the present proof-of-principle study, we demonstrate both (a) orbit selection by means of optical imaging at the insertion device beamline (IDB) of the MLS during TRIB operation and (b) its application for eTOF spectroscopy which adds a new quality for users of compact storage rings. In Fig. 1(a), the standard orbit (orange) is illustrated in comparison to a specific TRIB orbit closing after three turns (different shades of blue) which may be stored simultaneously. ${ }^{13}$ In general, both orbits are transversely separated in space and/or angle. Thus, at certain cuts perpendicular to the beam direction, one obtains simultaneously four source spots, one of the standard orbit and three of the threefold TRIB orbit, as shown in Fig. 1(b). The image in Fig. 1(c) was obtained while the regular standard
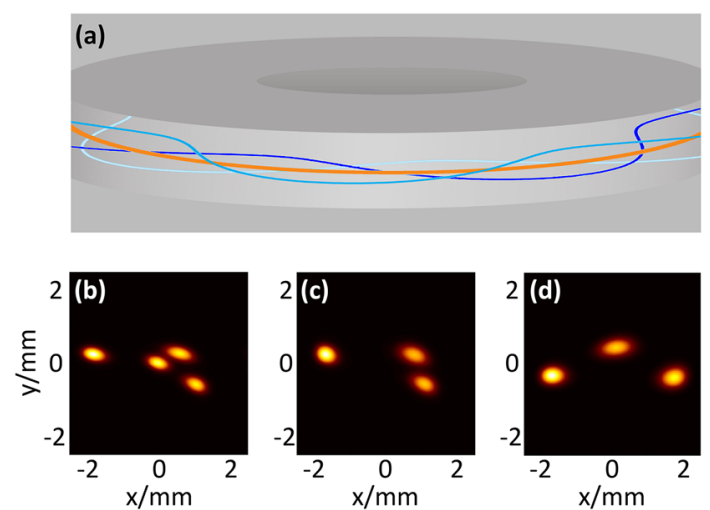

FIG. 1. (a) Sketch of the TRIB orbit at the MLS closing after three turns (different shades of blue), twisting around the standard orbit (orange), i.e., being transversely separated in space and angle. (The gray hollow cylinder symbolizes the storage ring). (b) Cross section of the electron beam during TRIB orbit single bunch operation of the MLS measured by using a source spot imaging system. (c) The same as (b), however, with an empty regular standard orbit in the centre (at $\mathrm{x}=\mathrm{y}=0$ position). (d) The same as (c), however, by using another source spot imaging system at a different position along the storage ring. 13 orbit was not populated so that only the three source spots of the threefold TRIB orbit are visible and that in Fig. 1(d) was obtained by means of another source spot imaging system at a different position along the storage ring and a different spatial source spot distribution.

In the configuration described, i.e., in the threefold TRIB orbit mode with an empty central orbit, the present measurements were performed at the insertion device beamline (IDB) of the MLS which is sketched in Fig. 2(a). IDB utilizes photons from the MLS undulator with $125 \mathrm{~mm}$ period length (U125) in the photon energy range from $1.5 \mathrm{eV}$ to $285 \mathrm{eV}$ by means of a normal incidence-grazing incidence (NI-GI) hybrid monochromator with the source spot being imaged onto the exit slit plane. In order to select one of the three source spots of the TRIB orbit and suppress the other two as much as possible, the exit slit and different beamline apertures were used in combination with a proper adjustment of the pre-mirror. The spatial source spot discrimination achieved at IDB is demonstrated in Fig. 2(b) which shows the relative photon flux behind the exit slit (at the photon energy of $31 \mathrm{eV}$ ) as measured with the help of a gas photoionization cell while scanning the horizontal deflection angle of the pre-mirror. Since the spatial distribution of the three TRIB spots at U125 corresponds almost to the image in Fig. 1(c), i.e., a good separation of one single spot from the other two in the horizontal (x) direction, the two maxima in Fig. 2(b) can be attributed accordingly, i.e., the single spot relates to the left peak and the other two to the broader peak at the right.

At the position marked in Fig. 2(b), i.e., with photons basically from one single source spot of the threefold TRIB orbit filled with one single electron bunch, measurements were performed with a linear eTOF spectrometer of the Scienta ARTOF 10k (Scienta Omicron, Uppsala, Sweden) type. ${ }^{18}$ By means of the IDB re-focus mirror Fig. 2(a), the photon beam was guided onto an $\mathrm{Au}(111)$ single crystal sample within a vacuum chamber equipped with the ARTOF and a second electron spectrometer, namely, an electrostatic hemispherical electron energy analyser of the Scienta R4000 (Scienta Omicron, Uppsala, Sweden) type. ${ }^{19}$ The two spectrometers were arranged in an orthogonal geometry and are facing the same interaction region on the sample. The sample surface was cleaned by performing repeatedly cycles of Ar ion sputtering and annealing at $650{ }^{\circ} \mathrm{C}$. The background pressure during the measurements was in the order of $7 \times 10^{-8} \mathrm{~Pa}$.

Figure 3 shows an ARTOF spectrum taken at the photon energy of $44 \mathrm{eV}$ employing, however, a so high bias voltage on the mesh in front of the ARTOF multi-channel plate (MCP) detector so that electrons were rejected. Thus, only photons scattered from the sample surface could reach the MCP. From the period of revolution at the MLS, one would expect, in the single bunch mode, every $160 \mathrm{~ns}$ a photon pulse which is usually too short for eTOF spectroscopy. However, the second and third peaks in Fig. 3 are strongly suppressed which reflects the almost complete discrimination of one single orbit (\#1) at IDB in the TRIB mode at the MLS with 5\% contribution from the other two orbits (\#2 and \#3) only. This contribution can be further reduced at the expense of photon flux. 
(a)

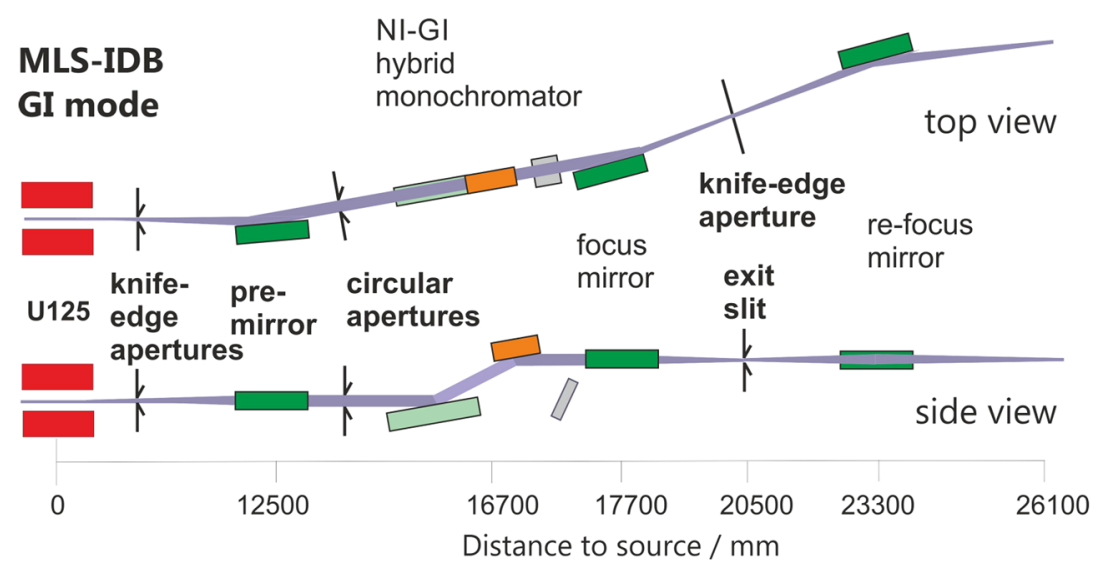

(b)

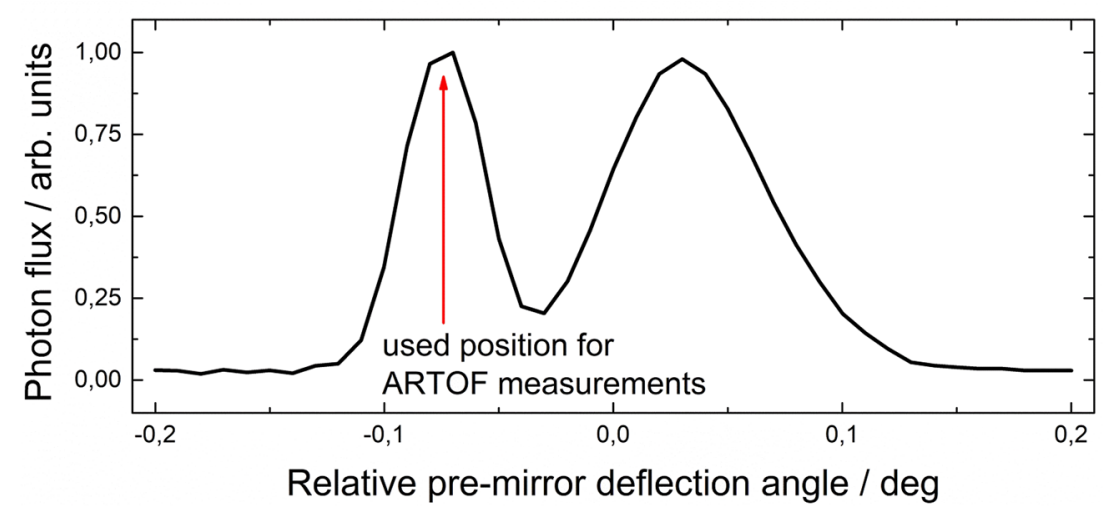

FIG. 2. (a) Sketch of the insertion device beamline (IDB) at the MLS combining a grazing incidence (GI) and a normal incidence (NI) monochromator. The source spot is imaged onto the plane of the exit slit. (b) Photon flux behind the exit slit as a function of the horizontal pre-mirror deflection angle relative to the standard position (set to 0 ).

Thus, an operational mode at the IDB/MLS was realized with a photon pulse interval of $3 \times 160 \mathrm{~ns}=480 \mathrm{~ns}$, i.e., sufficient for eTOF spectroscopy. At a ring current of below $1 \mathrm{~mA}$, it was used to measure eTOF spectra of the $\mathrm{Au}(111)$ sample using the ARTOF spectrometer. Figure 4(a) shows the spectrum of the $\mathrm{Au} 4 \mathrm{f}$ electrons obtained at the photon energy of $150 \mathrm{eV}$ and Fig. 4(b) shows that of the Au valence band obtained at $44 \mathrm{eV}$ (black upper curves), both compared, respectively, to results obtained with the R4000 analyser (red lower curves). The latter

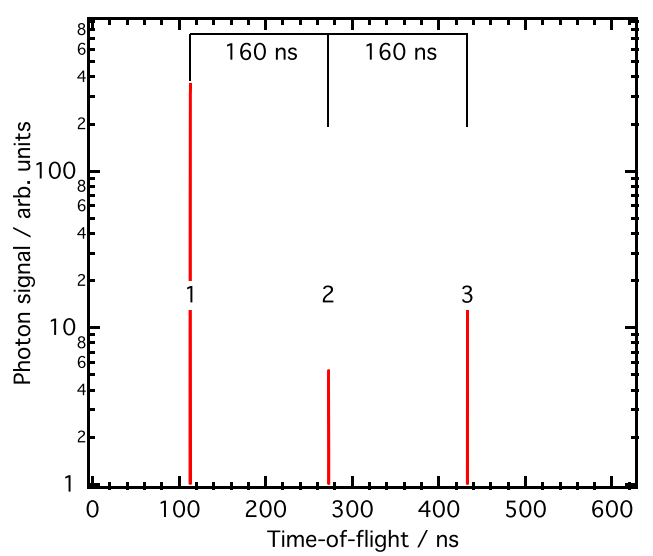

FIG. 3. Time-of-flight spectrum recorded with the ARTOF spectrometer using a high bias voltage on the detector mesh in order to reject electrons and accept photons scattered from the sample only. measurements were performed one day later, without breaking the vacuum or manipulating the sample, in the regular standard orbit multi-bunch operation mode of the MLS at a ring current beyond $100 \mathrm{~mA}$, however, at slightly reduced transmission and increased resolution of the IDB monochromator. Although the energy resolution of the ARTOF spectra is, therefore, a bit lower, the equivalence of the data sets obtained at comparable accumulation times is obvious. Taking into account the lower ring current and at least 10 times lower photon flux during the ARTOF measurements demonstrates the tremendous capability of eTOF spectrometers, i.e., by far higher responsivity due to the simultaneous detection of the complete electron energy spectrum compared to electrostatic analysers.

The $60 \mathrm{eV}$ electrons of Fig. 4(a) and the $35 \mathrm{eV}$ electrons of Fig. 4(b) refer to a time-of-flight within the ARTOF in the order of $200 \mathrm{~ns}$ to $300 \mathrm{~ns}$, respectively, i.e., longer than the single bunch period of revolution at the MLS of $160 \mathrm{~ns}$ but significantly shorter than the photon pulse interval of $480 \mathrm{~ns}$ of the new operational mode. Hence, selecting a single orbit by optical imaging of a threefold TRIB orbit makes eTOF spectroscopy feasible at the MLS or other compact SR sources with a circumference shorter than $100 \mathrm{~m}$.

In conclusion, two-orbit operation was realized at PTB's MLS by transverse resonance island buckets. Source spot selection was successfully demonstrated by means of optical imaging at the MLS insertion device beamline. The scheme might be of relevance for the upcoming BESSY VSR project 

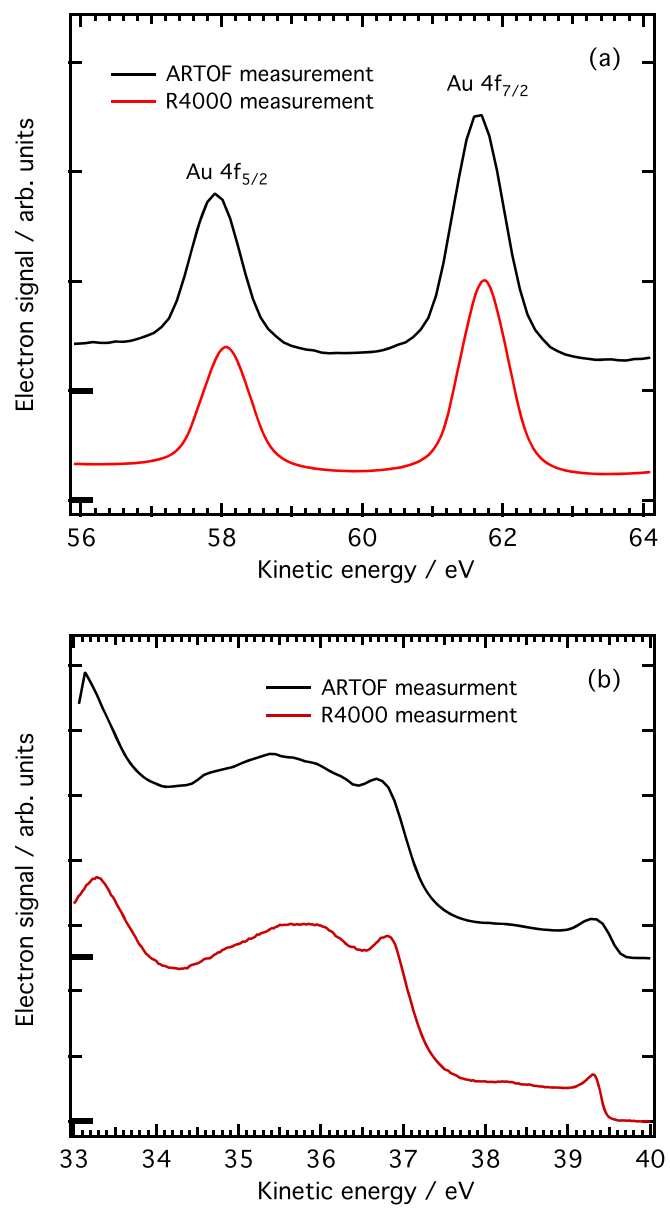

FIG. 4. Comparison of (a) the Au 4f electron energy spectra (at $150 \mathrm{eV}$ photon energy) and (b) Au valence band electron energy spectra (at $44 \mathrm{eV}$ photon energy) as recorded using the ARTOF spectrometer in the TRIB orbit single bunch operation mode of the MLS at a ring current of below $1 \mathrm{~mA}$ (black upper curves) and the R4000 analyser in the regular standard orbit multibunch operation mode of the MLS at a ring current beyond $100 \mathrm{~mA}$ (red lower curves).

in combination with SR beamline upgrades further optimized for multiple orbit and source spot discrimination. Moreover, a specific second orbit closing after three turns, which is based on transverse resonance island buckets, was tested. A photon pulse interval three times longer than the single bunch period of revolution qualifies the MLS for the application of highly efficient electron time-of-flight spectroscopy which is of significant importance for PTB's future programs of angular-resolved electron spectroscopy with synchrotron radiation together with external partners ${ }^{20-25}$ and similar projects at compact storage rings in general.

${ }^{1}$ W. Ackermann et al., Nat. Photonics 1, 336 (2007).

${ }^{2}$ S. D. Mitri et al., Proc. IPAC 2011, 918.

${ }^{3}$ P. Emma et al., Nat. Photonics 4, 641 (2010).

${ }^{4}$ T. Shintake et al., Nat. Photonics 2, 555 (2008).

${ }^{5}$ D. Pile, Nat. Photonics 5, 456 (2011).

${ }^{6}$ E. Allaria et al., Nat. Photonics 6, 699 (2012).

${ }^{7}$ E. A. Schneidmiller, B. Faatz, M. Kuhlmann, J. Rönsch-Schulenburg, S. Schreiber, M. Tischer, and M. V. Yurkov, Phys. Rev. Accel. Beams 20, 020705 (2017).

${ }^{8}$ P. F. Tavares, S. C. Leemann, M. Sjöström, and Å. Andersson, J. Synchrotron Radiat. 21, 862 (2014).

${ }^{9}$ A. Jankowiak et al., "BESSY VSR technical design study," Techical Report No. 1, Helmholtz-Zentrum Berlin f. Materialien und Energie GmbH, 2016.

${ }^{10}$ A. Gottwald, R. Klein, R. Müller, M. Richter, F. Scholze, R. Thornagel, and G. Ulm, Metrologia 49, S146 (2012).

${ }^{11}$ J. Feikes, M. von Hartrott, M. Ries, P. Schmid, G. Wüstefeld, A. Hoehl, R. Klein, R. Müller, and G. Ulm, Phys. Rev. Spec. Top: Accel. Beams 14, 030705 (2011).

${ }^{12}$ M. Ries, "Nonlinear momentum compaction and coherent synchrotron radiation at the metrology light source," Ph.D. thesis, Humboldt-Universität zu Berlin, Mathematisch-Naturwissenschaftliche Fakultät I, 2014.

${ }^{13}$ M. Ries et al., Proc. IPAC 2015, 138.

${ }^{14}$ R. W. Schoenlein, S. Chattopadhyay, H. H. W. Chong, T. E. Glover, P. A. Heimann, C. V. Shank, A. A. Zholents, and M. S. Zolotorev, Science 287, 2237 (2000).

${ }^{15}$ K. Holldack, S. Khan, R. Mitzner, and T. Quast, Phys. Rev. Lett. 96, 054801 (2006).

${ }^{16}$ K. Holldack, R. Ovsyannikov, P. Kuske, R. Müller, A. Schälicke, M. Scheer, M. Gorgoi, D. Kühn, T. Leitner, S. Svensson, N. Mårtensson, and A. Föhlisch, Nat. Commun. 5, 4010 (2014).

${ }^{17}$ C. Sun, G. Portmann, M. Hertlein, J. Kirz, and D. S. Robin, Phys. Rev. Lett. 109, 264801 (2012).

${ }^{18}$ R. Ovsyannikov, P. Karlsson, M. Lundqvist, C. Lupulescu, W. Eberhardt, A. Föhlisch, S. Svensson, and N. Mårtensson, J. Electron Spectrosc. Relat. Phenom. 191, 92 (2013).

${ }^{19}$ G. Öhrwall, P. Karlsson, M. Wirde, M. Lundqvist, P. Andersson, D. Ceolin, B. Wannberg, T. Kachel, H. Dürr, W. Eberhardt, and S. Svensson, J. Electron Spectrosc. Relat. Phenom. 183, 125 (2011).

${ }^{20}$ F. Roth, M. Herzig, C. Lupulescu, E. Darlatt, A. Gottwald, M. Knupfer, and W. Eberhardt, J. Appl. Phys. 118, 185310 (2015).

${ }^{21}$ S. Weiß, D. Lüftner, T. Ules, E. M. Reinisch, H. Kaser, A. Gottwald, M. Richter, S. Soubatch, G. Koller, M. G. Ramsey, F. S. Tautz, and P. Puschnig, Nat. Commun. 6, 8287 (2015).

${ }^{22}$ F. Roth, C. Lupulescu, E. Darlatt, A. Gottwald, and W. Eberhardt, J. Electron Spectrosc. Relat. Phenom. 208, 2 (2016).

${ }^{23}$ E. Darlatt, B. Muhsin, R. Roesch, C. Lupulescu, F. Roth, M. Kolbe, A. Gottwald, H. Hoppe, and M. Richter, Nanotechnology 27, 324005 (2016).

${ }^{24}$ D. Lüftner, S. Weiß, X. Yang, P. Hurdax, V. Feyer, A. Gottwald, G. Koller, S. Soubatch, P. Puschnig, M. G. Ramsey, and F. S. Tautz, Phys. Rev. B 96, 125402 (2017).

${ }^{25}$ F. Roth, T. Arion, H. Kaser, A. Gottwald, and W. Eberhardt, J. Electron Spectrosc. Relat. Phenom. 224, 84 (2018). 